\title{
Calculation of pressure fields from arbitrarily shaped, apodized, and excited ultrasound transducers
}

Jensen, Jørgen Arendt; Svendsen, Niels Bruun

Published in:

I E E E Transactions on Ultrasonics, Ferroelectrics and Frequency Control

Link to article, DOI:

$10.1109 / 58.139123$

Publication date:

1992

Document Version

Publisher's PDF, also known as Version of record

Link back to DTU Orbit

Citation (APA):

Jensen, J. A., \& Svendsen, N. B. (1992). Calculation of pressure fields from arbitrarily shaped, apodized, and excited ultrasound transducers. I E E E Transactions on Ultrasonics, Ferroelectrics and Frequency Control, 39(2), 262-267. https://doi.org/10.1109/58.139123

\section{General rights}

Copyright and moral rights for the publications made accessible in the public portal are retained by the authors and/or other copyright owners and it is a condition of accessing publications that users recognise and abide by the legal requirements associated with these rights.

- Users may download and print one copy of any publication from the public portal for the purpose of private study or research.

- You may not further distribute the material or use it for any profit-making activity or commercial gain

- You may freely distribute the URL identifying the publication in the public portal 


\title{
Calculation of Pressure Fields from Arbitrarily Shaped, Apodized, and Excited Ultrasound Transducers
}

\author{
Jørgen Arendt Jensen and Niels Bruun Svendsen
}

\begin{abstract}
A method for the simulation of pulsed pressure fields from arbitrarily shaped, apodized and excited ultrasound transducers is suggested. It relies on the Tupholme-Stepanishen method for calculating pulsed pressure fields, and can also handle the continuous wave and pulse-echo case. The field is calculated by dividing the surface into small rectangles and then summing their response. A fast calculation is obtained by using the farfield approximation. Examples of the accuracy of the approach and actual calculation times are given.
\end{abstract}

\section{INTRODUCTION}

$\mathbf{T}$ HE MOST IMPORTANT component in acquiring high quality images for medical ultrasound scanners is the probing transducer. Ultimately it determines the quality of the data acquired, and thus the quality of the images and parameters displayed. Considerable effort has therefore been spend on designing transducers and characterizing the field emitted and received [1], [2].

Several methods for calculating the pressure field have been developed for assisting in the design and characterization of various transducer geometries. Most of the methods can be traced to the fundamental solutions of Rayleigh, King, and Schoch, of which a review can be found in [3].

The most powerful approach seems to be the method developed by Tupholme and Stepanishen [4]-[6], which gives an exact solution for a transducer modeled as a planar piston vibrating uniformly in an infinite rigid, planar baffle. Analytic expressions for several transducer types have been found [5], [7], but closed form solutions can not be found for all types. Especially the introduction of exotic geometries or apodization of the transducer surface leads to analytically unsolvable integrals.

In this paper we will develop a simulation approach based on the Tupholme-Stepanishen approach, which can simulate transducers with any apodization of the transducer surface and with any excitation of the transducer.

Manuscript received June 12, 1991; revised October 21, 1991; accepted October 22, 1991. This work was supported in part by the Danish Technical Research Council, with grant $16-4218$, in part by Brüel and $\mathrm{Kjær} \mathrm{A} / \mathrm{S}$, in part by Novo's Foundation, in part by H.C. Ørsted's Foundation, and in part by Trane's Foundation.

J. A. Jensen is with the Electronics Institute, Building 349, Technical University of Denmark, 2800 Lyngby, Denmark, and he is currently visiting the Department of Biomedical Engineering, Duke University, Durham, NC 27706

N. B. Svendsen is with the Danish Acoustical Institute, Akademivej, Building 356, DK-2800 Lyngby, Denmark.

IEEE Log Number 9105552
The paper proceeds along the following lines. Section II formulates the problem and details the underlying theory. Section III gives various implementation details and Section IV lists a number of examples for different transducer geometries and apodization functions. It will be shown that the method is fast and gives accurate answers.

\section{THEORY}

The purpose of this paper is to devise a fast and accurate method for calculating the pulsed pressure field emitted from an arbitrarily shaped, apodized, and excited ultrasound transducer

It is assumed that the transducer is mounted in an infinite, rigid baffle. Enforcing appropriate boundary conditions, the emitted field can be found by solving the wave equation for the velocity potential $\psi[4],[5]$ :

$$
\nabla^{2} \psi-\frac{1}{c_{0}^{2}} \frac{\partial^{2} \psi}{\partial^{2} t}=0
$$

from which the pressure is calculated as:

$$
p_{1}(\vec{r}, t)=\rho_{0} \frac{\partial \psi(\vec{r}, t)}{\partial t}
$$

where $\rho_{0}$ is the mean density of the media, $c_{0}$ is the propagation velocity, and $p_{1}$ is the over pressure.

The coordinate system shown in Fig. 1 is used in the calculation. The particle velocity normal to the transducer surface is denoted by $v\left(\vec{r}_{2}+\vec{r}_{3} . t\right)$. The solution to the homogeneous wave equation using Greens function is [5]

$\psi\left(\vec{r}_{1}, \vec{r}_{2}, t\right)=\int_{S} \int_{0}^{t} v\left(\vec{r}_{2}+\vec{r}_{3}, t_{2}\right) g\left(\vec{r}_{1}, t \mid \vec{r}_{2}+\vec{r}_{3}, t_{2}\right) d t_{2} d^{2} \vec{r}_{3}$

where $S$ denotes the transducer surface. $g$ is the time-dependent Green's function and is

$$
g\left(\vec{r}_{1}, t \mid \vec{r}_{2}+\vec{r}_{3}, t_{2}\right)=\frac{\delta\left(t-t_{2}-\frac{\left|\vec{r}_{1}-\vec{r}_{2}-\vec{r}_{3}\right|}{c_{0}}\right)}{2 \pi\left|\vec{r}_{1}-\vec{r}_{2}-\vec{r}_{3}\right|}
$$

where $\left|\vec{r}_{1}-\vec{r}_{2}-\vec{r}_{3}\right|$ is the distance from the surface to the point where the field is calculated The integral is a statement of Huygens' principle, that for a planar vibrating surface each point on the source generates a spherical wave, and the resulting field is found by integrating these waves at 


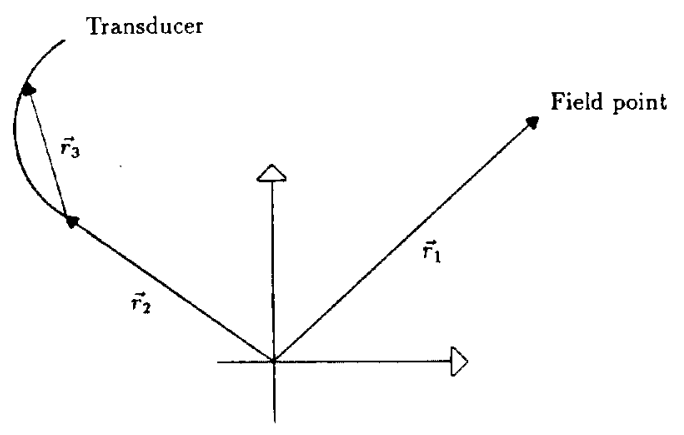

Fig. 1. Coordinate system for calculating the incident field.

the point of interest under the assumption of radiation into an isotropic, homogeneous, nondissipative medium.

If a slightly curved transducer is used, an additional term is introduced as shown in Morse and Feshbach [8]. This term is called the second order diffraction term in Penttinen and Luukkala [7]. It can be shown to vanish for a planar transducer, and as long as the transducer is only slightly curved and large compared to the wavelength of the ultrasound, the resulting expression is a good approximation to the pressure field [7].

If it is assumed that surface vibration accounting for the excitation function and electromechanical impulse response can be split into a spatial component $a\left(\vec{r}_{2}+\vec{r}_{3}\right)$ and a temporal component $v_{e}\left(t_{2}\right)$ then:

$$
\begin{aligned}
\psi\left(\vec{r}_{1}, \vec{r}_{2}, t\right)=\int_{0}^{t} \int_{S} v_{\epsilon}\left(t_{2}\right) a\left(\vec{r}_{2}+\vec{r}_{3}\right) \\
\cdot g\left(\vec{r}_{1}, t \mid \vec{r}_{2}+\vec{r}_{3}, t_{2}\right) d^{2} \vec{r}_{3} d t_{2}
\end{aligned}
$$

where $a(\vec{r})$ is denoted the spatial source velocity distribution [10]. This implies that the vibration amplitude at a certain point on the surface does not depend on time, so the amplitude of vibration is not influenced by the shape of the excitation.

The function

$$
h_{a}\left(\vec{r}_{1}, \vec{r}_{2}, t-t_{2}\right)=\int_{S} a\left(\vec{r}_{2}+\vec{r}_{3}\right) g\left(\vec{r}_{1}, t \mid \vec{r}_{2}+\vec{r}_{3}, t_{2}\right) d^{2} \vec{r}_{3}
$$

is called the apodized spatial impulse response and it relates the transducer geometry to the acoustical field. By this function we can write

$$
\psi\left(\vec{r}_{1}, \vec{r}_{2}, t\right)=v_{e}(t) \underset{t}{\star} h_{a}\left(\vec{r}_{1}, \vec{r}_{2}, t\right)
$$

where $v_{e}(t)$ is the piston velocity waveform, and the velocity potential is written as a convolution in time between this and the apodized spatial impulse response.

If the particle velocity is assumed to be uniform over the surface of the transducer, (5) can be reduced to [6]:

$$
\psi\left(\vec{r}_{1}, \vec{r}_{2}, t\right)=\int_{0}^{t} v_{e}\left(t_{2}\right) \int_{S} g\left(\vec{r}_{1}, t \mid \vec{r}_{2}+\vec{r}_{3}, t_{2}\right) d^{2} \vec{r}_{3} d t_{2}
$$

where the last integral equals the traditional spatial impulse response.

Note that $h_{a}$ depends on the difference between $\vec{r}_{1}$ and $\vec{r}_{2}$, thus it is spatially varying. To emphasize this $h_{a}$ is written $h_{a}\left(\vec{r}_{1}, \vec{r}_{2}, t\right)$.
The sound pressure for the incident field then is

$$
p\left(\vec{r}_{1}, \vec{r}_{2}, t\right)=\rho_{0} \frac{\partial \psi\left(\vec{r}_{1}, \vec{r}_{2}, t\right)}{\partial t}=\rho_{0} v_{e}(t) \underset{t}{\star} \frac{\partial h_{a}\left(\vec{r}_{1}, \vec{r}_{2}, t\right)}{\partial t}
$$

or

$$
p\left(\vec{r}_{1}, \vec{r}_{2}, t\right)=\rho_{0} \frac{\partial v_{e}(t)}{\partial t} \star h_{a}\left(\vec{r}_{1}, \vec{r}_{2}, t\right)
$$

Note here the separation between the excitation and the transducer geometry. The $v_{e}(t)$ includes the electromechanical impulse response of the transducer [2].

Explicit solutions for a number of transducer geometries have been found. Analytical expressions for the circular, flat transducer can be found in [5], and for the circular, concave geometry in [7], [9].

It must be emphasized that only two approximations are used here. The first is the assumption of a large and slightly curved transducer, and the second assumption is that of separability between excitation and transducer geometry. Transducers can be constructed in which this is a very good approximation, so that the pressure field calculated by this method is in good agreement with the measured field.

The geometric features of the transducer are contained in the apodized spatial impulse response $h_{a}\left(\vec{r}_{1}, \vec{r}_{2}, t\right)$ and from this the field for any excitation function, including the continuous wave case, can be calculated. Further, it has been shown that the pulse echo field received by the emitting transducer can be calculated by [11]:

$$
\begin{aligned}
p_{r}\left(\vec{r}_{2}, t\right) & =\frac{1}{c_{0}^{2}} \frac{\partial^{2} v_{p e}(t)}{\partial t^{2}} \stackrel{\star}{t} H_{p e}\left(\vec{r}_{1}, \vec{r}_{2}, t\right) \\
& =\frac{1}{c_{0}^{2}} \frac{\partial^{2} v_{p e}(t)}{\partial t^{2}} \stackrel{\star}{t} h\left(\vec{r}_{1}, \vec{r}_{2}, t\right) \stackrel{\star}{t} h\left(\vec{r}_{2}, \vec{r}_{1}, t\right)
\end{aligned}
$$

where $v_{p e}$ is the pulse-echo electromechanical impulse response including the excitation function. So the emitted pulsed field, the received field, and the continuous wave case can be derived from the apodized spatial impulse response as the electromechanical impulse response usually can be determined from a simple measurement.

Thus, the original problem is transformed into calculating the apodized spatial impulse response. Closed form solutions have been found for some cases as mentioned previously, but not for all geometries and rarely when apodization is used.

\section{A. Simulation Method}

In analytic calculations the solution is found by evaluating which part of a sphere with center at the field point that intersects the transducer surface [9]. The area of the strip on the radiator surface divided by the distance to the field point gives the spatial impulse response at that time instance in the case of uniform vibration. When apodization is used, the different areas on the strip should be suitably weighted.

In this simulation method the problem is reversed. A spherical wave is emitted from a point on the aperture and all spherical waves are summed at the field point. weighted by the inverse of the distance from the aperture point to the 
field point. The apodized spatial impulse response is then approximated by

$$
h_{a}\left(\vec{r}_{1}, \vec{r}_{2}, t\right) \approx \sum_{S} a\left(\vec{r}_{2}+\vec{r}_{i}\right) \frac{\delta\left(t-\frac{\vec{r}_{1}-\vec{r}_{2}-\vec{r}_{2}}{\left.c_{0}\right)}\right.}{\left|\vec{r}_{1}-\vec{r}_{i}-\vec{r}_{2}\right|}
$$

where $r_{i}$ denotes the points on the transducer surface.

Just dividing the surface into points has the drawback that quite a large number of points must be used as the variance of $h_{a}$ at a time instance depends on the number of spherical waves received in one time interval. The number of points can be drastically reduced by dividing the surface into small rectangles and then summing the responses from these rectangles. This is the approach used here. A similar approach has also been studied by Ocheltree and Frizzel for the continuous wave case [12]. Here we are studying the pulsed field case in which the continuous wave case can be calculated as a special case.

Dividing the transducer surface into squares introduces an approximation to the true geometry, and the field will deviate from the true one. The problem is reduced by using small squares, where the distance to the field point is large compared to the size of the squares. Thus, it is appropriate to use a farfield approximation, when calculating the contribution from each individual element. The exact solution for the impulse response from a rectangular piston is derived in [6] so only an intuitive explanation for the far-field solution is given here. As the impulse response at a point in front of a piston is proportional to how large part of the piston that contributes to the response at a given time, the problem of deriving the response is reduced to geometric considerations concerning the distance between the field point and the different parts of the transducer. From a point near the piston surface the isodistance curves looks like shown in Fig. 2(a), but if the distance increases the curves tends to straight lines, which is shown in Fig 2(b). The first is the near-field situation and the latter is the far-field situation.

To calculate the far-field response from the rectangle a description of the piston and the location of the field point as shown in Fig. 3 is needed. The piston is described by its length and width and to fix the location of the field point, the piston is placed in a coordinate system in the $X Y$-plane with the center at the origin. Then the location is defined by the field point's position vector, split up into a unit vector $\left(x_{e}, y_{e}, z_{e}\right)$ and a distance, $l$.

In general the far-field spatial impulse response has the shape of a trapezoid as shown in Fig. 4, where $t_{1}$ is the timeof-flight from the nearest corner of the piston to the field point. Likewise $t_{2}$ and $t_{3}$ are the time-of-flight from the second and third nearest corner, and $t_{4}$ is the time-of-flight from the corner with the largest distance to the field point. In special cases two or more of the $t$ 's are equal.

The trapezoid shape response can be calculated by convolving two rectangular pulses. The width of these pulses are calculated by projecting the length and width of the piston onto the line through the rectangles center and the field point.

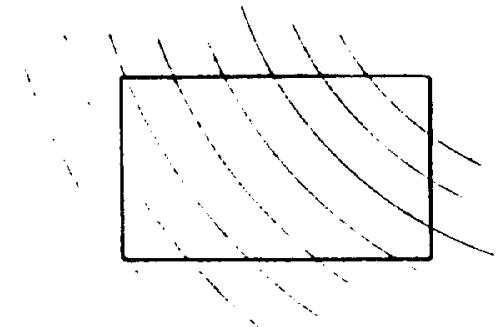

(a)

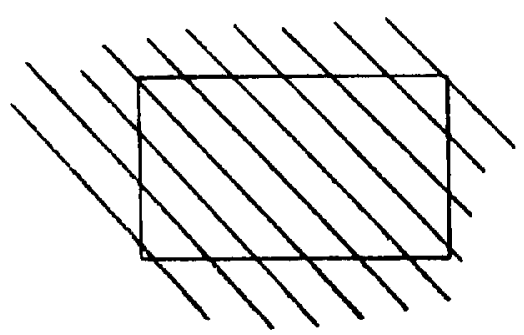

(b)

Fig. 2. Isodistance curves on the transducer surface. (a) Near-field. (b) Far-field.

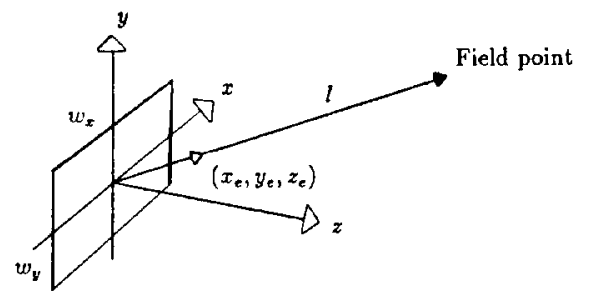

Fig. 3. Description of the piston and its orientation.

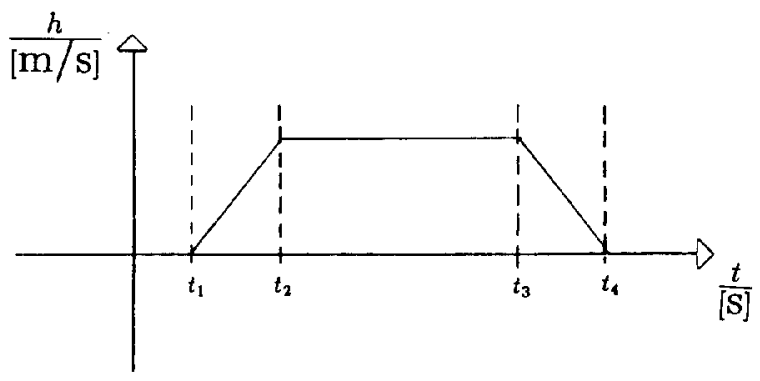

Fig. 4. Far-field response.

Based on the mentioned description of the system we get

$$
\begin{aligned}
& \Delta t_{1}=\min \left(\frac{w_{y} \cdot y_{e}}{c_{0}}, \frac{w_{x} \cdot x_{e}}{c_{0}}\right) \\
& \Delta t_{2}=\max \left(\frac{w_{y} \cdot y_{e}}{c_{0}}, \frac{w_{x} \cdot x_{e}}{c_{0}}\right)
\end{aligned}
$$

where $w_{y}$ and $w_{x}$ are the side lengths of the rectangle.

The arrival times are then calculated by

$$
\begin{aligned}
& t_{1}=\frac{l}{c_{0}}-\frac{\Delta t_{1}+\Delta t_{2}}{2} \\
& t_{2}=t_{1}+\Delta t_{1} \\
& t_{3}=t_{1}+\Delta t_{2}
\end{aligned}
$$




$$
t_{4}=t_{1}+\Delta t_{1}+\Delta t_{2}
$$

The amplitude of the trapezoid is a function of piston area and distance to the field point. The shape depends on $\Delta t_{1}$ and $\Delta t_{2}$, but the area of the trapezoid integrated over the whole time interval is always equal to

$$
a_{r e c}(l)=\frac{w_{x} \cdot w_{y}}{2 \cdot \pi \cdot l}
$$

\section{B. Arbitrarily Shaped Piston}

This quite simple result for the far-field response of a rectangular piston can be used when designing a fast numerical method for calculating the response of a piston of any shape. As for the point source representation the piston is split up into small squares (or rectangles), but instead of representing the small areas by point sources the far-field response from the rectangles is used. This makes it possible to use larger and therefore fewer squares to describe the piston. The resulting response is calculated by summing up the responses from all the squares. Apodizing is obtained by multiplying the individual responses by an apodizing factor, that could be a function of e.g. the radius of the transducer. In a similar manner a time delay can be added to the response, giving a different phase for different parts of the transducer, like in a phased array.

\section{Far-Field Region}

The size of the rectangles must be chosen so that the field point lies in the far-field region. This is given by [1]:

$$
l \gg \frac{w^{2}}{4 \lambda}
$$

where $l$ is the distance to the field point, $w$ the largest dimension of the rectangle and $\lambda$ the wavelength, which equals $c_{0} / f$, where $\mathrm{f}$ is frequency. If $f$ is the highest frequency in the response simulated, the side length should obey the relation

$$
w \ll \sqrt{4 l c_{0} / f} .
$$

Examples indicating how close the side length can be chosen to this limit and what accuracy then is obtained, are given in Section IV.

\section{OVERView of Program}

The prime application of this program is to investigate fields from transducers of shapes with unknown analytic solutions and to study the influence of apodization and phasing of elements. It is very difficult to imagine the shape of these fields, and therefore whether correct results are calculated. In order to solve this problem, the program has been divided into two parts. The first calculates the position and orientation of the small rectangles describing the transducer, and the second performs the field calculation. The two parts are independent thereby enabling the possibility of thoroughly testing and calibrating the field calculation, which is transducer independent. The only uncertainty is then the placement of the rectangles. This part of the program can, however, be interfaced to a CAD program, that can visualize the placement

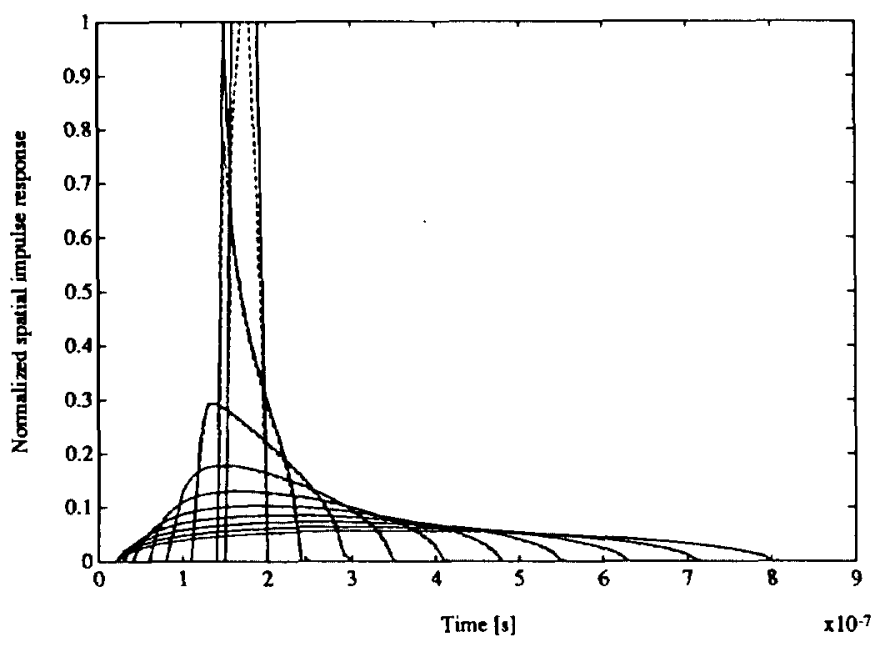

Fig. 5. Simulated (-) and true (- - spatial impulse response of concave transducer. The time on the $y$-axis is relative. Zero corresponds to $t=T \tau .83 \mu \mathrm{s}$.

of the rectangles. By this method accurate and reliable results should be assured.

\section{EXAMPLES}

In this section several examples of use of the program are shown. Responses are compared to analytic solutions and guidelines for choosing the number of elements and the resulting computing times are given.

The first example is for a concave, nonapodized transducer with an aperture radius of $8 \mathrm{~mm}$ and a focal distance of 150 $\mathrm{mm}$. An analytic expression is found for this geometry [9] and can thus be compared to simulated responses.

The spatial impulse response at a distance of $120 \mathrm{~mm}$ from the surface is shown in Fig. 5 from on the acoustical axis and out in steps of $1 \mathrm{~mm}$. The transducer was divided into 3177 squares with a side length of $0.25 \mathrm{~mm}$. Calculating the ten lines of the spatial impulse response at a sampling frequency of 100 $\mathrm{MHz}$ took $1.5 \mathrm{~s}$ on an HP/Apollo 9000/425t workstation ${ }^{1}$, and is shown as dashed lines in Fig. 5. We see that the program quite accurately tracks the theoretical spatial impulse response off the acoustical axis. On the axis it is, however, more difficult to get the exact position and shape of the abrupt changes in the spatial impulse response due to the employment of the far-field approximation.

In the next example the concave transducer was apodized with a Gaussian distribution function defined as

$$
a(r)=e^{-a_{p}^{2}\left(\frac{r}{R}\right)^{2}}
$$

where $R$ is the radius of the aperture and $r$ the distance from the center. $a_{p}$ was chosen to be 2 . The spatial impulse response is shown in Fig. 6. The characteristic elimination of sharp edges in the spatial impulse response is seen.

To show that the pulse-echo response can be calculated to good accuracy a single example is shown in Fig. 7. The measured and simulated responses were obtained at a distance

\footnotetext{
${ }^{1}$ This workstation has roughly the calculation speed of a $40-\mathrm{MHz} 486 \mathrm{PC}$.
} 


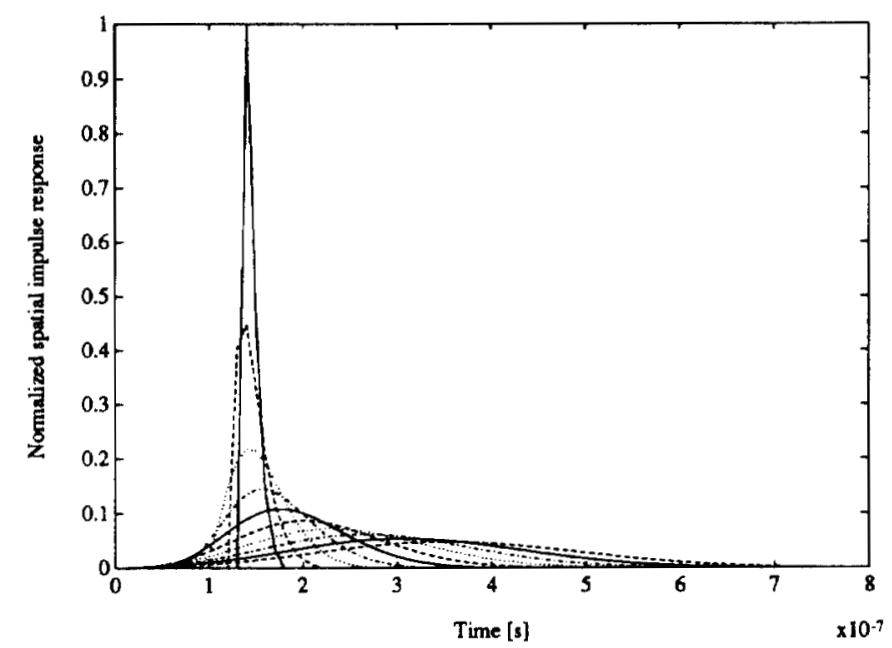

Fig. 6. Simulated spatial impulse response of Gaussian apodized concave transducer. The time on the $y$-axis is relative. Zero corresponds to $t=7.8 .83 \mu \mathrm{s}$.

of $120 \mathrm{~mm}$ from the transducer surface. The measured pressure field was acquired by moving a needle in steps of $0.2 \mathrm{~mm}$ with an accuracy of $0.006 \mathrm{~mm}$, measuring in a plane containing the acoustical axis of the transducer. Before the measurement, the transducer and the needle were aligned so that the needle was parallel to the acoustical axis. The data were sampled at a frequency of $100 \mathrm{MHz}$. The simulated field was calculated by measuring $v_{p e}$ as the response from a planar reflector, and then using (11) to calculate the field. The simulation was done with a element size of $0.25 \mathrm{~mm}$, and the calculation took 12.1 seconds.

The envelope of the RF-signals is shown as a contour plot with $6 \mathrm{~dB}$ between the contours. The plots span $20 \mathrm{~mm}$ in the lateral direction and $4 \mu \mathrm{s}$ in the axial direction. An other example at $60 \mathrm{~mm}$ from the transducer surface is shown in Fig. 8, indicating the good agreement between simulation and measurement. Further details and examples can be found in [11].

An important parameter to be selected is the element size, which essentially determines the accuracy of the result. The size should depend on the distance to the field, as this determines how well the far-field approximation is. In Table I the quantity $\left(w^{2} f /\left(4 l c_{0}\right)\right)$ is shown against the normalized mean square error (MSE) defined by

$$
\text { MSE }=\sqrt{\frac{\sum_{i=1}^{N}\left(h_{\text {cal }}(i)-h_{\text {true }}(i)\right)^{2}}{\sum_{i=1}^{N} h_{\text {true }}^{2}(i)}}
$$

where $N$ is the number of samples in the responses.

The figures shown were calculated for the concave, nonapodized transducer mentioned previously at $120 \mathrm{~mm}$ from the surface and $10 \mathrm{~mm}$ off the acoustical axis at a sampling frequency of $100 \mathrm{MHz}$. Also shown in the table is the calculation time and calculation time multiplied by the number of Flops obtained in the Linpack benchmark and divided by the number of elements (NCT). All experiments were conducted on an HP/Apollo 9000/425t with a Linpack rating of $1.8 \mathrm{Mflop}$. Using the last number in the table a rough estimate of the

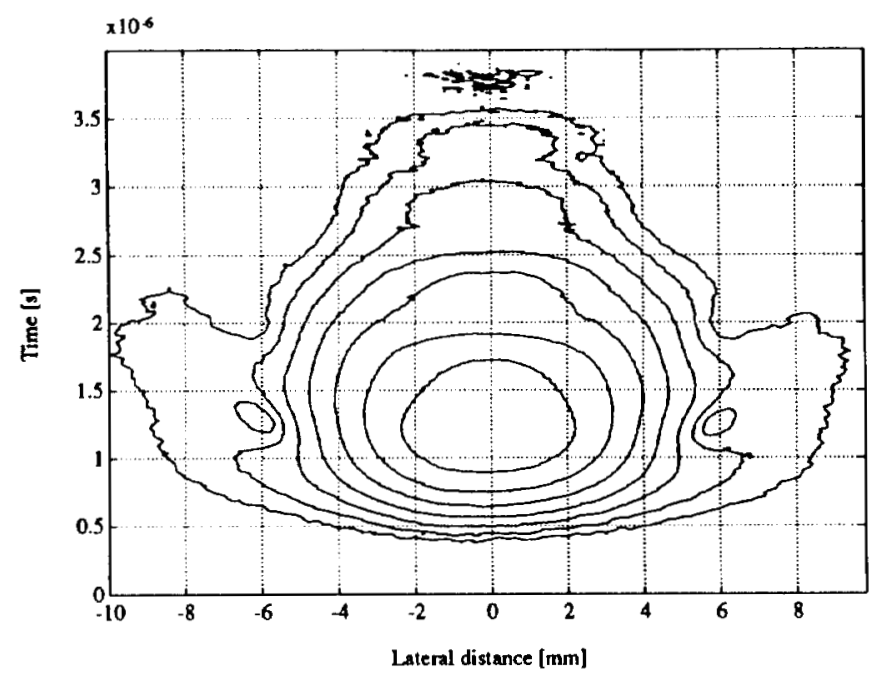

(a)

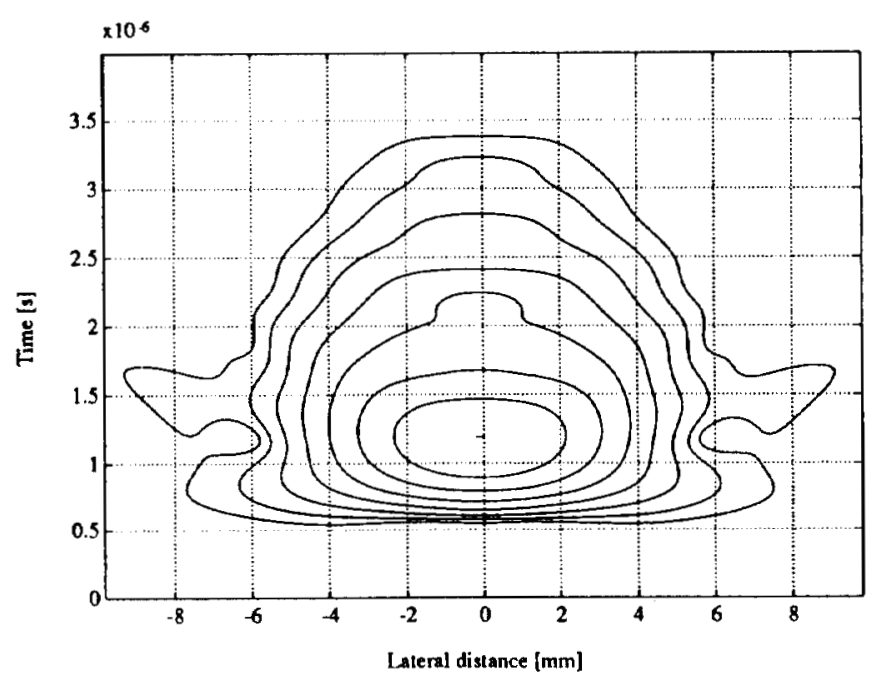

(b)

Fig. 7. Measured and simulated pulse-echo response for a concave transducer. Response at $x=120 \mathrm{~mm}$. The time on the $y$-axis is relative. Zero corresponds to $t=77.83 \mu \mathrm{s}$.

calculation time on other computers can be determined from:

$$
T=\frac{\text { NCT } \cdot N_{\text {squares }}}{L_{\text {in }}}
$$

where $N_{\text {squares }}$ is the number of elements and $L_{\text {in }}$ the Linpack rating. So for the newer HP/Apollo 730 workstation with a Linpack rating of 22 MFlops, it would take $0.11 \mathrm{~s}$ to calculate the response with $0.1-\mathrm{mm}$ rectangles.

\section{CONCLUSION}

A method for the calculation of fields from arbitrarilyshaped and apodized transducers has been given. A calculation time in the order of a few seconds were obtained by splitting the transducer into small squares, and summing their far-field responses.

Any excitation of the transducer can be handled and the continuous wave solution found by Fourier transforming the calculated responses. It was also shown how to obtain the 


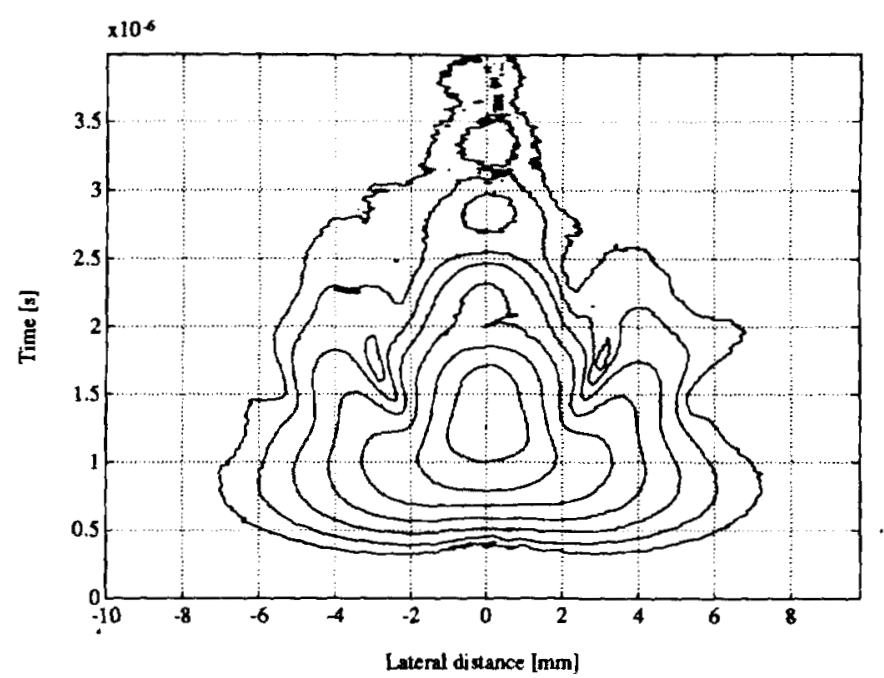

(a)

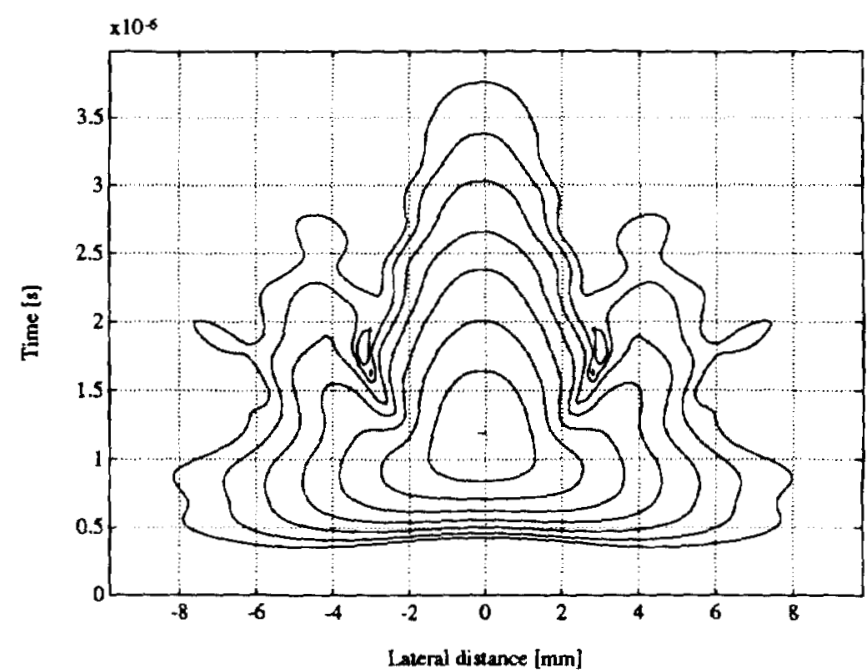

(b)

Fig. 8. Measured and simulated pulse-echo response for a concave transducer. Response at $x=60 \mathrm{~mm}$. The time on the $y$-axis is relative. Zero corresponds to $t=38.92 \mu \mathrm{s}$.

TABLE

TABle OVER accuracy and Computation Time for Different Element Sizes

\begin{tabular}{|c|c|c|c|c|c|}
\hline $\begin{array}{l}\text { No. of } \\
\text { elements }\end{array}$ & $\begin{array}{l}\text { Side } \\
\text { length }\end{array}$ & $\frac{u^{2} f}{4 l_{0}}$ & $\begin{array}{l}\mathrm{MSE}^{\mathrm{a}} \\
\%\end{array}$ & $\begin{array}{l}\text { Calculation } \\
\text { time (s) }\end{array}$ & $\mathrm{NCT}^{\mathrm{b}}$ \\
\hline 788 & $0.5 \mathrm{~mm}$ & 0.0169 & 14.7 & 0.1 & 233.77 \\
\hline 3177 & $0.25 \mathrm{~mm}$ & 0.0042 & 6.4 & 0.3 & 173.93 \\
\hline 20046 & $0.1 \mathrm{~mm}$ & $6.76 \cdot 10^{-4}$ & 3.5 & 1.4 & 128.64 \\
\hline
\end{tabular}

MSE is mean square error (see text).

${ }^{b} \mathrm{NCT}$ the normalized calculation time (see text).

pulse-echo field, so all characteristics of the field can be found by this simulation method.

The accuracy of the approach is on the order of 3 to 5 percent compared to the theoretical spatial impulse response (see (19)), when a reasonable number of elements is used ensuring short calculation times. During the work with the program, it was found that the main cause for the deviations to the true response, was the use of the far-field approximation rather than the geometric approximation of using squares. We therefore hope to improve the accuracy and still attain short calculation time by improving on this approximation in future work.

\section{ACKNOWLEDGMENT}

Klaus Bolding Rasmussen is thanked for his help during the development of the technique and its implementation. Dr. Ole Trier Andersen is thanked for valuable discussions during the preparation of the paper.

\section{REFERENCES}

[1] G. S. Kino, Acoustic waves, Devices, imaging \& analog signal processing. Englewood Cliffs, NJ: Prentice Hall, 1987.

[2] J. W. Hunt, M. Arditi, and F. S. Foster, "Ultrasound transducers for pulse-echo medical imaging," IEEE Trans. Biomed. Eng., vol. BME-30, no. 8, pp. $453-481$, Aug. 1983

[3] G. R. Harris, "Review of transient field theory for a baffled planar piston," J. Acoust. Soc. Am., vol 70, pp. 10-20, 1981.

[4] G. E. Tupholme, "Generation of acoustic pulses by baffled plane pistons," Mathematika vol. 16, pp. 209-224, 1969.

[5] P. R. Stepanishen, "The time-dependent force and radiation impedance on a piston in a rigid infinite planar baffle," J. Acoust. Soc. Am., vol 49 , no. 3 , pp. $841-849,1971$.

[6] _- "Transient radiation from pistons in a infinite planar baffle," $J$ Acoust. Soc. Am. vol. 49, pp. 1627-1638, 1971.

[7] A. Penttinen and M. Luukkala, "The impulse response and pressure nearfield of a curved ultrasonic radiator," J. Phys. D., vol, 9, pp. 1547-1557, 1976.

(8] P. M. Morse and H. Feshbach, Methods of Theoretical Physics, Part I. New York: McGraw-Hill, 1953.

[9] M. Arditi, F. S. Forster and J. Hunt, "Transient fields of concave annular arrays," Ultrason. Imaging, vol. 3, pp. 37-61, 1981.

[10] G. R. Harris, "Transient field of a baffled planar piston having an arbitrary vibration amplitude distribution," J. Acoust. Soc. Am., vol. 70 , pp. 186-204, 1981.

[11] J. A. Jensen, "A model for the propagation and scattering of ultrasound in tissue," I. Acoust. Soc. Am., vol. 89, no. 1, pp. 182-191, 1991.

[12] K. B. Ocheltree and L. A. Frizzel, "Sound field calculation for rectangular sources," IEEE Trans. Ultrason., Ferroelec., Freq. Contr., vol. 36, no. 2, pp. 242-248, Mar. 1989.

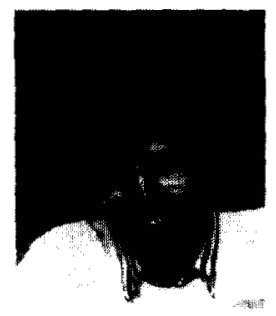

Jorgen Arendt Jensen was born in Roskilde, Denmark, in 1960. He received the M.Sc. degree in electrical engineering in 1985 from the Technical University of Denmark. He received the Ph.D. degree in 1989 from the Technical University. The subject of his Ph.D. was digital signal processing of medical ultrasound images.

He is a Visiting Scientist at the Department of Biomedical Engineering, Duke University, Durham, NC. He currently holds a postdoctoral fellowship a the Technical University and has published a num ber of reports and papers on digital signal processing, ultrasound acoustics, and medical uitrasound imaging.

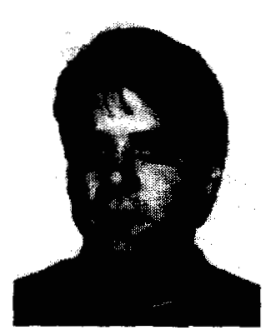

Niels Bruun Svendsen was born in Copenhagen, Denmark, in 1962. He received the B.Sc. degree in electrical engineering in 1986 from the Engineering Academy of Denmark.

He has been with the Danish Acoustical Institute since 1986 as a Staff Scientist, where he has worked with computer algorithms in the fields of sound propagation, siren coverage planning, and noise and vibration analysis. In 1987 he carried out a study on coherent Doppler simulation at TNO Institute of Applied Physics, Delft, The Netherlands. Currently, he is engaged in the development of a noise analysis system. 